\title{
Clinical Characteristics of Kawasaki Disease in Markazi Province, Iran
}

\author{
Yazdan Ghandi ${ }^{1,{ }^{*}}$, Danial Habibi ${ }^{2}$, Manigeh Kahbazi ${ }^{3}$, Fatemeh Dorreh ${ }^{4}$ and Maryam Lotfi ${ }^{5}$ \\ ${ }^{1}$ Department of Pediatric Cardiology, Arak University of Medical Sciences, Arak, Iran \\ ${ }^{2}$ Department of Biostatistics, Arak University of Medical Sciences, Arak, Iran \\ ${ }^{3}$ Department of Pediatric Infection, Amirkabir Hospital, School of Medicine, Arak University of Medical Sciences, Arak, Iran \\ ${ }^{4}$ Department of Pediatrics, School of Medicine, Arak University of Medical Sciences, Arak, Iran \\ ${ }^{5}$ Amirkabir Hospital, School of Medicine, Arak University of Medical Sciences, Arak, Iran \\ "Corresponding author: Associate Professor of Pediatric Cardiology, Department of Pediatric Cardiology, Arak University of Medical Sciences, Arak, Iran. Email: \\ drghandi1351@gmail.com
}

Received 2018 October 23; Accepted 2019 November 01.

\begin{abstract}
Background: Kawasaki disease (KD) is described as a life-threatening vasculitis, which mostly develops in children below five years of age and is diagnosed according to clinical criteria. It is also a common rheumatologic disorder in Iran.

Objectives: The present study aimed at determining the clinical and demographic characteristics of KD patients in Iran.

Methods: We retrospectively assessed 69 cases of KD in an Iranian pediatric population from March 2014 to March 2018. The Japanese Kawasaki Disease Research Committee guidelines were used as the diagnostic criteria for typical KD. Incomplete or atypical KD was diagnosed in patients with coronary artery changes, but without all the criteria for KD.

Results: In this study, 69 patients were recruited, with a male-female ratio of 1:8. Overall, $64 \%$ (n, 44) and 36\% of children met the criteria for typical and atypical KD, respectively. Also, echocardiographic abnormalities were reported in eight patients (12\%). Coronary artery aneurysm was found in $2 \%$ of patients, while other cardiac abnormalities were found in $12 \%$ of patients. The male-female ratio of coronary artery anomalies and other cardiac abnormalities was 3:1. Polymorphic exanthema was the most common clinical manifestation. The erythrocyte sedimentation rate $\geq 40 \mathrm{~mm} / \mathrm{h}$ was the most common laboratory finding, while skin desquamation was the most common complication in other organs.

Conclusions: KD is not uncommon in Markazi Province, Iran. In this study, distribution of demographic characteristics was not similar to reports from other countries. Also, clinical findings, age and gender distribution, laboratory findings, and complications in other organs were not similar to previous reports. The incidence of typical and atypical KD was different in this region, especially in terms of complications, such as cardiac and gastrointestinal complications.
\end{abstract}

Keywords: Epidemiology, Children, Iran, Kawasaki Disease, Vasculitis

\section{Background}

Kawasaki disease (KD) is described as an acute vasculitis of unknown etiology, which occurs in all populations (1, 2 ). In developed countries, KD is the most important cause of acquired cardiovascular disease in children. It mostly affects children under five years of age ( $80 \%)$, and the average age of disease onset is two to three years $(1,2)$. In addition, men are more prone to KD than women (male-female ratio, 1.5:1)(3).

For the first time, KD was described by Kawasaki in 1967 according to the clinical characteristics reported in 50 Japanese children $(1,2)$. It was defined as a distinctive disease, associated with fever, oropharyngeal mucosal changes (e.g., strawberry tongue and fissured lips), bilateral conjunctival injection without exudate, polymorphous exanthem, swelling or redness of the feet and hands, cervical lymphadenitis, and erythema of the palms and soles. Besides persistent fever, five clinical features are fundamental to the diagnosis of KD: erythema of pharyngeal and oral mucosa, cervical lymphadenopathy, bilateral nonexudative bulbar conjunctivitis, hand and foot edema, and rashes.

In classic KD, diagnosis is established if the patient has persistent fever for five days or longer and presents with at least four out of five diagnostic criteria for KD. Alternatively, atypical or incomplete KD is diagnosed when the patient has persistent fever, but less than four out of five diagnostic criteria. It is known that laboratory and echocardiographic findings may improve diagnosis in atypical cases (1-4).

Some laboratory findings are associated with KD and may contribute to its diagnosis: thrombocytosis, anemia, elevated erythrocyte sedimentation rate (ESR), leukocyto- 
sis, and positive C-reactive protein (CRP). In addition, coronary artery abnormalities are observed in approximately one-quarter of untreated children. According to previous studies, coronary artery aneurysm (CAA), which is the most severe consequence of $\mathrm{KD}$, has been reported in $20 \%-25 \%$ of patients without treatment (5-7).

\section{Objectives}

The present study aimed at increasing the current knowledge of demographic and clinical features of KD patients at the pediatric ward of Amir-Kabir Children's Hospital in Markazi province, Iran.

\section{Methods}

In this descriptive cross-sectional study, patients with $\mathrm{KD}$, hospitalized in the pediatric ward of Amir-Kabir Children's Hospital in Arak, were recruited from January 2013 to July 2018. The study protocol was approved by the Research Ethics Committee of Arak medical University (IR.ARAKMU.REC.1397.205). Prior to the study, written informed consents were obtained from the parents of children.

Children with the diagnostic signs and symptoms of $\mathrm{KD}$, including a five-day fever plus four out of five major clinical features of KD (i.e., classic KD), were recruited. In addition, children with fever, along with two or three major clinical features of KD and echocardiographic results indicative of coronary vessel involvement (i.e., atypical KD), were included.

The diagnostic criteria for KD were in line with the guidelines of the Japanese Kawasaki Disease Research Committee. Accordingly, atypical KD was diagnosed in children with coronary artery abnormalities, who did not strictly meet all the diagnostic criteria. For all subjects, echocardiographic evaluation was performed and repeated in the first two months. Clinical manifestations, laboratory results, cardiac and non-cardiac sequelae of the disease, and demographic and clinical characteristics of patients, including age, gender, type of treatment, onset of intravenous immunoglobulin (IVIG) or aspirin therapy, and duration of response to treatment, were carefully documented.

The participants were examined in terms of the diagnostic criteria, echocardiographic results, surrogate markers, and treatment interventions. Questionnaires were used to gather data concerning the patient's age at disease onset, gender, clinical features, duration and presence of fever along with other diagnostic criteria, laboratory findings (e.g., high platelet count, low hemoglobin, high ESR, and positive CRP), echocardiographic results, treatment features, follow-up, and outcomes.

In the first two weeks, the patients were administered IVIG ( $2 \mathrm{~g} / \mathrm{kg})$ and an anti-inflammatory dose of aspirin (100 $\mathrm{mg} / \mathrm{kg} /$ day), followed by methylprednisolone pulses and antiplatelet doses of aspirin (3-5 mg/kg/day) for the following six weeks.

\subsection{Statistical Analyses}

The statistical parameters are expressed as frequency and percentage. For data analysis, SPSS software version 25 was used.

\section{Results}

During six years, 69 children were diagnosed with KD in the present study. Nearly all subjects were younger than six years at disease onset. The mean age of children was $33.49 \pm 24.31$ months, and the age range was 7-120 months; the majority of children were two years old (Table 1). Also, 45 (65.2\%) patients were male, and 24 (34.8\%) were female (male-female ratio, 1.8:1).

Overall, 44 (64\%) patients met four or five KD criteria, 12 (17\%) met four criteria, 8 (12\%) met three criteria, and 5 (7\%) met two criteria. In addition, $81 \%$ of patients were diagnosed in the first ten days. Table 2 indicates the laboratory findings and clinical manifestations of the patients. The body temperature of $83 \%$ of children was above $38.8^{\circ} \mathrm{C}$. The mean fever duration was 9.56 days, which indicates no significant difference between the non-cardiac and cardiac groups $(\mathrm{P}>0.05)$.

Based on the findings, the mean interval between the onset of symptoms and diagnosis was 7.23 days; however, the longest time to establish a diagnosis was 38 days. The time needed to confirm the diagnosis was different between the cardiac and non-cardiac groups (median, 9.7 days vs. 6.9 days), although it was insignificant $(\mathrm{P}>0.05)$. Moreover, the mean interval between the disease onset and development of desquamation was 16.74 days. No positive

\begin{tabular}{ll}
\hline Table 1. The Patients' Age at the Onset of Disease & \\
\hline Age (months) & No $(\%)$ \\
\hline$\leq \mathbf{1 2}$ & $15(21.7)$ \\
$\mathbf{1 3 - 2 4}$ & $19(27.6)$ \\
$\mathbf{2 5}-\mathbf{3 6}$ & $11(15.9)$ \\
$\mathbf{3 7 - 4 8}$ & $7(10.2)$ \\
$\mathbf{4 9 - 6 0}$ & $7(10.2)$ \\
$\mathbf{6 1 - 7 2}$ & $8(11.6)$ \\
$\geq \mathbf{7 3}$ & $2(2.8)$ \\
\hline
\end{tabular}




\begin{tabular}{|c|c|c|}
\hline Clinical Features & Positive & Negative \\
\hline Fever & $69(100)$ & $0(0)$ \\
\hline Polymorphic exanthema & $38(55)$ & $31(45)$ \\
\hline Bilateral conjunctiva injection & $32(46)$ & $37(54)$ \\
\hline Changes in the oral cavity and lips & $29(42)$ & $40(58)$ \\
\hline Changes in extremities & $24(35)$ & $45(65)$ \\
\hline Agitation & $22(32)$ & $47(68)$ \\
\hline Cervical lymphadenopathy & $21(30)$ & $48(70)$ \\
\hline \multicolumn{3}{|c|}{ Laboratory Findings } \\
\hline $\mathrm{ESR} \geq 40 \mathrm{~mm} / \mathrm{h}$ & $52(75)$ & $17(25)$ \\
\hline CRP $\geq 3.0+$ & $42(61)$ & $27(39)$ \\
\hline Abnormal liver function test & $34(49)$ & $35(51)$ \\
\hline Hypoalbuminemia $\leq 3.0 \mathrm{~g} / \mathrm{dL}$ & $22(32)$ & $47(68)$ \\
\hline Hyponatremia & $22(32)$ & $47(68)$ \\
\hline $\begin{array}{l}\text { Urine } \geq 10 \mathrm{WBC} / \text { high-power field (sterile } \\
\text { pyuria) }\end{array}$ & $11(16)$ & $59(84)$ \\
\hline Urine $\geq 5$ RBC/high-power field & $5(7)$ & $64(93)$ \\
\hline \multicolumn{3}{|c|}{ Involvement of Other Organs } \\
\hline Desquamation & $52(75)$ & $17(25)$ \\
\hline Cardiac involvement & $8(12)$ & $61(88)$ \\
\hline Arthralgia & $7(10)$ & $62(90)$ \\
\hline Aseptic meningitis & $3(4)$ & $66(96)$ \\
\hline Hydrops of the gallbladder & $2(3)$ & $67(97)$ \\
\hline BCG inoculation site & $2(3)$ & $67(97)$ \\
\hline
\end{tabular}

cultures (i.e., blood, urine, and throat) were identified in our patients. Table 3 show the involvement of other organs in KD patients. The seasonal distribution of KD showed in Figure 1.

Coronary artery anomalies and other arterial abnormalities were reported in $8(12 \%)$ patients, including six males and two females. A nine-month-old girl and a sixyear-old boy developed giant aneurysms with a lumen diameter of $>8 \mathrm{~mm}$. The echocardiographic results were reported in four patients with classic $\mathrm{KD}(6 \%)$ and 25 patients with atypical $\mathrm{KD}(36 \%)$. In the latter group, 15 patients met three out of five clinical criteria for KD, and ten patients only met two out of five criteria.

The echocardiographic findings indicated a definitive aneurysm in one patient, perivascular edema in two patients, pericardial effusion in one patient, and myocarditis along with mitral regurgitation in four patients. CAA

\begin{tabular}{|c|c|}
\hline Laboratory Findings & Positive, No. (\%) \\
\hline \multicolumn{2}{|c|}{ Leukocytosis $\left(\mathrm{WBC} / \mathrm{mm}^{3}\right)$} \\
\hline$\geq 15000$ & $20(29)$ \\
\hline$\geq 25000$ & $4(6)$ \\
\hline \multicolumn{2}{|c|}{ Absolute neutrophil (count/mm³) } \\
\hline$\geq 2500$ & $7(10)$ \\
\hline$\geq 5000$ & $38(55)$ \\
\hline$\geq 10000$ & $21(30)$ \\
\hline \multicolumn{2}{|l|}{ ESR } \\
\hline$\geq 40$ & $40(74)$ \\
\hline$\geq 100$ & $14(26)$ \\
\hline \multicolumn{2}{|l|}{ CRP } \\
\hline$\geq 3+$ & $15(22)$ \\
\hline$\geq 4+$ & $5(7)$ \\
\hline \multicolumn{2}{|c|}{ Thrombocytosis (platelet $>450000 / \mathrm{mm}^{3}$ ) } \\
\hline$\geq 450000$ & $40(74)$ \\
\hline$\geq 1000000$ & $11(16)$ \\
\hline SGOT elevation & $23(33)$ \\
\hline SGPT elevation & $21(30)$ \\
\hline \multicolumn{2}{|l|}{ Hemoglobin } \\
\hline$<10 \mathrm{~g} / \mathrm{dL}$ & $10(14)$ \\
\hline$<12 \mathrm{~g} / \mathrm{dL}$ & $7(10)$ \\
\hline \multicolumn{2}{|l|}{ Pyuria } \\
\hline$\geq 10$ & $10(14)$ \\
\hline$\geq 20$ & $4(6)$ \\
\hline
\end{tabular}

Abbreviations: ESR, erythrocyte sedimentation rate; SGOT, serum glutamic oxaloacetic transaminase; SGPT, serum glutamic-pyruvic transaminase.

occurred in one patient (2\%), which persisted for one year. Overall, 25 (36\%) children received antibiotics for their disease, as they did not meet the KD criteria. Two patients were treated with methylprednisolone.

\section{Discussion}

KD is described as a vasculitis of unknown etiology, which mostly involves children. However, no specific tests are available for the diagnosis of $\mathrm{KD}(1-3)$. Therefore, certain clinical criteria should be fulfilled to confirm the diagnosis (4). Based on the Japanese guidelines for KD diagnosis, four or five out of five major clinical criteria for KD should be identified in patients.

The clinical characteristics of KD are not pathognomonic. Therefore, to confirm a diagnosis, other diseases, which mimic its clinical features (i.e., toxin-mediated 


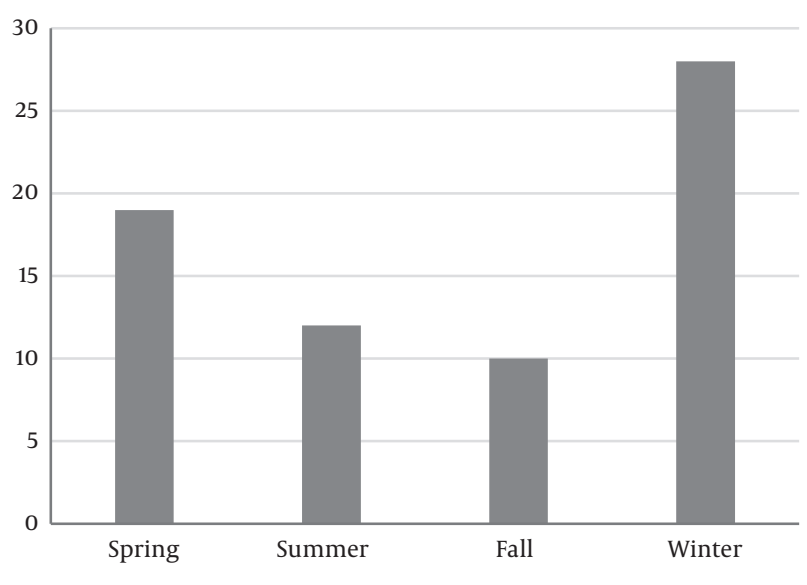

Figure 1. The seasonal distribution of KD

staphylococcal and streptococcal diseases, systemic allergic reactions to medications, and infections with enterovirus, adenovirus, or measles), should be excluded. Nevertheless, the clinical course of classic KD is well characterized (8-10).

The main objective of our study was to identify the clinical presentations, epidemiology, laboratory results, and cardiac sequelae of KD in Markazi Province, Iran. Considering the age distribution of $69 \mathrm{KD}$ patients, the majority were clustered in the 13- to 24-month-old age group. The mean age of children was $33.49 \pm 24.31$ months at diagnosis. In addition, the majority of patients were born in winter months. This seasonal trend has been also reported in previous studies, in which KD patients were prominently born in February (winter) (11).

Three patients showed recurrence and presented with coronary artery disease. The risk of prolonged fever and thrombocytosis was significantly higher in these patients, and they were considered as possible risk factors for coronary artery disease. The present findings are concurrent with a study by Asadi-Pooya et al. on $113 \mathrm{KD}$ patients in Shiraz, Iran. They also reported that prolonged fever and thrombocytosis are responsible for cardiac complications (12).

In our study, a total of 69 suspected patients were enrolled. Forty-four patients (64\%) were diagnosed with typical KD, and 25 (36\%) patients were diagnosed with atypical KD (male-female ratio, 1.8:1). In this regard, Shamsizadeh et al. showed that $64(61.5 \%)$ and $40(38.5 \%)$ patients met the diagnostic criteria for typical and atypical KD, respectively. In contrast to our study, fever, along with conjunctivitis and oral changes, was a cardinal sign (11). We found fever in $100 \%$ of our patients, followed by polymorphic exanthema. Lymphadenopathy was the least common clini- cal feature, mostly found unilaterally in the course of disease $(13,14)$.

Asadi-Pooya et al. examined 113 patients over 12 years. Similar to previous studies, a male preponderance was reported (male-female ratio, 2.1:1). The incidence of coronary artery disease was also higher in males. These findings considering the role of gender are in good agreement with our results. Also, in the study by Asadi-Pooya et al., the mean age of children was 3.89 years (12). Overall, $70 \%$ of patients were 1 - 5 years old, whereas $90 \%$ of our patients were younger than five years.

Asadi-Pooya et al. reported a median body temperature of $39.4^{\circ} \mathrm{C}$ in patients; based on their findings, temperature was above $39^{\circ} \mathrm{C}$ in $70 \%$ of patients. Also, the mean fever duration was 9.14 days, and no significant difference was found between the cardiac and non-cardiac groups ( $\mathrm{P}$ $>0.05$ ). On the other hand, in our study, the mean fever duration was 9.56 days, and body temperature was above $38.8^{\circ} \mathrm{C}$ in $83 \%$ of patients.

The mean interval between the onset of symptoms and disease diagnosis was seven days, and the maximum time needed to establish a diagnosis was 45 days. Diagnosis was confirmed within the first ten days in $75 \%$ of patients. The required time for confirming a diagnosis was different between the cardiac and non-cardiac groups (median, 10 days vs. 7 days); however, the difference was insignificant $(\mathrm{P}>$ $0.05)$. This finding is comparable with the present results.

Asadi-Pooya et al. (12) reported that $94.6 \%$ of patients presented with conjunctivitis, $93.8 \%$ with oral changes, $51.3 \%$ with extremity changes, $80.5 \%$ with rashes, $65.4 \%$ with cervical lymphadenopathy, and $41.5 \%$ with periungual desquamation; these clinical findings are in contradiction with our results. The main clinical finding of our study was polymorphous exanthema, and the least common clinical feature was cervical lymphadenopathy.

In contrast to our study, $25 \%$ of patients showed an ESR $>80 \mathrm{~mm} / \mathrm{h}$ in the study by Asadi-Pooya and colleagues. The median ESR was $64 \mathrm{~mm} / \mathrm{h}$ in the cardiac group, which is not significantly different from the non-cardiac group ( $\mathrm{P}$ $>0.05$ ) (12). We found an ESR of $>100 \mathrm{~mm} / \mathrm{h}$ in $26 \%$ of patients and an elevated ESR of $>40 \mathrm{~mm} / \mathrm{h}$ in $52(75 \%)$ patients. Unlike a previous study, which reported a CRP of 3 - 4 in 30\% of patients (15), our study found a CRP of $3-4+$ in 42 (61\%) patients. With regard to other laboratory findings, leukocytosis $\geq 15000 / \mathrm{mm}^{3}$ was reported in $24(35 \%)$ patients, with the predominance of polymorphonuclear cells. Also, thrombocytosis (platelet $>450000 / \mathrm{mm}^{3}$ ) was observed in 51 (90\%) patients, and anemia (hemoglobin level $\leq 12$ ) was reported in 17 (24\%) patients.

Siadati and Sabouni reported atypical KD in 36 out of 85 patients (42.3\%), with a male-female ratio of 1:36 (15). The major clinical findings observed in KD patients were 
changes in lips and mouth (70\%), strawberry tongue (58\%), lip fissures (50\%), skin eruptions as maculopapular rash (56\%), conjunctivitis without discharge (45\%), and changes in extremities (49\%). Similar to earlier studies, cervical lymphadenopathy (both bilateral and painless) was the least common finding among major clinical criteria for KD (43\%). Cardiac complications were found in $21 \%$ of patients, and aneurysms were identified in 7.3\% (15). Other clinical manifestations included gastrointestinal symptoms, such as diarrhea, vomiting, ileus, and icterus. Our findings do not support their observations, as polymorphous exanthema $(55 \%)$ was the main clinical finding in our study, cardiac complications were reported in $12 \%$ of patients, and aneurysms were found in $2 \%$.

Our results also revealed that 22 (32\%) children with KD were generally irritable and uncomfortable. High spiking fever was common, exceeding $38^{\circ} \mathrm{C}$ in nearly all patients. Periungual desquamation occurred in 52 (75\%) children two to three weeks following the fever onset. The majority of clinical manifestations in our patients were observed in the first two weeks of the disease onset. Cardiac complications were reported in 8 (12\%) children towards the second to fourth week of the disease (16).

Cardiovascular complications, as the most important contributors to morbidity and mortality in patients with $\mathrm{KD}$, primarily emerge in the acute phase. Cardiac involvements include pericardial effusion, myocardial inflammation (in up to $50 \%$ of patients), and coronary artery abnormalities, which usually develop after ten days of the disease onset. Coronary artery disorders (aneurysm or ectasia) have been reported in $20 \%$ - $25 \%$ of untreated patients. Other cardiac findings, i.e., CAA, acute myocarditis, congestive heart failure, mitral insufficiency, arterial ectasia, and pericarditis with pericardial effusion, were more common in the present study, compared to earlier research $(5,6,10)$.

Asadi-Pooya et al. (12) showed that among ten patients with cardiac involvement, three had coronary aneurysms in their primary echocardiography, and seven presented with dilated $(>4 \mathrm{~mm})$ coronary arteries. In addition, two cases showed pericardial effusion. Six patients showed tricuspid regurgitation, and all ten patients showed mitral regurgitation. In four patients, dilated coronary arteries regressed almost after four weeks, based on the second echocardiography; this condition remained in three patients for at least two months.

In the current study, the prevalence of cardiac involvement was $12 \%$, which is slightly different from previous reports $(20 \%)(15,17)$. The prevalence of CAA in our study $(2 \%)$ was consistent with reports from other countries. However, a study from Isfahan, Iran, reported a higher incidence of CAA (22.6\%), compared to other reports (18). In their study, male gender, WBC count $>20000$ per $\mathrm{mm}^{3}$, elevated ESR, pericardial effusion, and lack of IVIG treatment in the first ten days of the disease were found responsible for coronary aneurysms; careful follow-up was recommended in these patients (18). In our study, aneurysms only developed in one patient, who was administered IVIG for treatment before the tenth day of KD development.

Many other manifestations of KD, including arthritis, indicate its systemic nature. Arthritis often involves small finger and toe joints and is more common among girls than boys $(8-10,15)$. In our study, arthralgia was observed in $7(10 \%)$ patients, and aseptic meningitis was identified in $3(4 \%)$ patients. Also, urethritis with sterile pyuria was reported in $11(16 \%)$ patients. Mild hepatitis with an increased level of transaminases was reported in 44 cases. In addition, hydrops of the gallbladder occurred in 2 (3\%) patients, leading to severe pain in the right upper quadrant.

In order to manage KD, use of aspirin and IVIG as antiinflammatory drugs and long-term anticoagulation are suggested. Treatment with IVIG and aspirin could majorly change KD management. There are controversies about the ideal time for initiating IVIG treatment, although it is not a major concern to emergency physicians. Generally, treatment is more successful if initiated in the first ten days after the fever onset.

The risk of CAA increases due to delayed diagnosis or treatment, which is more common in older children. If a child meets all the clinical criteria for KD, IVIG treatment is almost certain, particularly if surrogate markers confirm the diagnosis. The classic dose of IVIG is a single daily infusion of $400 \mathrm{mg} / \mathrm{kg} / \mathrm{d}$ for four days or a single infusion of 2 $\mathrm{g} / \mathrm{kg}$ for 12 hours. In the first two weeks, aspirin is recommended at high doses of $80-100 \mathrm{mg} / \mathrm{kg} / \mathrm{d}$ PO QID, followed by maintenance doses of $3-5 \mathrm{mg} / \mathrm{kg}$ PO QID for six to eight weeks. Reinfusion is necessary at $2 \mathrm{~g} / \mathrm{kg}$ if fever persists or recurs two days after the initial IVIG infusion. Sometimes, multiple infusions of IVIG are used, especially in severe KD cases.

A group of KD patients, who show resistance to IVIG treatment, is the most vulnerable group to CAA and longterm sequelae of the disease. In some of our patients, these manifestations were reported. Despite timely IVIG treatment, $5 \%$ of children with KD remain at risk of CAA. In our study, the incidence of cardiac complications was $12 \%$. IVIG is still the gold standard treatment for the prevention of CAA; however, additional infusions and steroids or immunosuppressants may be necessary in 10\% - 15\% of children.

\subsection{Conclusions}

In this study, the demographic characteristics of patients were different from reports from other countries. The prevalence of atypical KD was higher among children, 
and there was a male predilection. Conversely, the prevalence of coronary artery anomalies or other cardiac disorders was lower.

\section{Footnotes}

Authors' Contribution: Study concept and design: Yazdan Ghandi, Fatemeh Dorreh, and Manizheh kahbazi; acquisition of data: Maryam Lotfi; analysis and interpretation of data: Danial Habibi; writing of the manuscript: Yazdan Ghandi and Danial Habibi.

Conflict of Interests: The authors declared no conflict of interests.

Ethical Approval: IR.ARAKMU.REC.1397.205.

Funding/Support: This study did not receive any specific grant from funding agencies in the public, commercial, or not-for-profit sectors.

\section{References}

1. Barron KS. Kawasaki disease in children. Curr Opin Rheumatol. 1998;10(1):29-37. doi: 10.1097/00002281-199801000-00005. [PubMed: 9448987]

2. Kawasaki T. [Acute febrile mucocutaneous syndrome with lymphoid involvement with specific desquamation of the fingers and toes in children]. Arerugi. 1967;16(3):178-222. Japanese. [PubMed: 6062087].

3. Burns JC, Glode MP. Kawasaki syndrome. Lancet. 2004;364(9433):53344. doi: 10.1016/S0140-6736(04)16814-1. [PubMed:15302199].

4. Rowley AH, Shulman ST. Kawasaki syndrome. Pediatr Clin North Am. 1999;46(2):313-29. doi: 10.1016/s0031-3955(05)70120-6. [PubMed: 10218077].

5. Hirata S, Nakamura Y, Matsumoto K, Yanagawa H. Long-term consequences of Kawasaki disease among first-year junior high school students. Arch Pediatr Adolesc Med. 2002;156(1):77-80. doi: 10.1001/archpedi.156.1.77. [PubMed: 11772195].

6. Iemura M, Ishii M, Sugimura T, Akagi T, Kato H. Long term consequences of regressed coronary aneurysms after Kawasaki disease: Vascular wall morphology and function. Heart. 2000;83(3):307-11. doi: 10.1136/heart.83.3.307. [PubMed: 10677411]. [PubMed Central: PMC1729327].
7. Melish ME, Marchette NJ, Kaplan JC, Kihara S, Ching D, Ho DD. Absence of significant RNA-dependent DNA polymerase activity in lymphocytes from patients with Kawasaki syndrome. Nature. 1989;337(6204):288-90. doi: 10.1038/337288a0. [PubMed: 2463487].

8. Newburger JW, Taubert KA, Shulman ST, Rowley AH, Gewitz MH, Takahashi M, et al. Summary and abstracts of the Seventh International Kawasaki Disease Symposium: December 4-7, 2001, Hakone, Japan. Pediatr Res. 2003;53(1):153-7. doi: 10.1203/00006450-200301000-00026. [PubMed: 12508096].

9. Rowley AH. Incomplete (atypical) Kawasaki disease. Pediatr Infect Dis J. 2002;21(6):563-5. doi: 10.1097/00006454-200206000-00015. [PubMed: 12182382].

10. Zhang T, Yanagawa H, Oki I, Nakamura Y, Yashiro M, Ojima T, et al Factors related to cardiac sequelae of Kawasaki disease. Eur J Pediatr. 1999;158(9):694-7. doi: 10.1007/s004310051181. [PubMed:10485297].

11. Shamsizadeh A, Ziaei Kajbaf T, Razavi M, Cheraghian B. Clinical and epidemiological characteristics of kawasaki disease. Jundishapur JMicrobiol. 2014;7(8). e11014. doi: 10.5812/jjm.11014. [PubMed: 25485046] [PubMed Central: PMC4255208].

12. Asadi-Pooya AA, Borzoee M, Amoozgar H. The experience with 113 patients with Kawasaki disease in Fars province, Iran. Turk J Pediatr. 2006;48(2):109-14. [PubMed: 16848108].

13. Fulton DR, Newburger JW. Long-term cardiac sequelae of Kawasaki disease. Curr Rheumatol Rep. 2000;2(4):324-9. doi:10.1007/s11926-0000070-2. [PubMed: 11123078]

14. Quasney MW, Bronstein DE, Cantor RM, Zhang Q, Stroupe C, Shike H, et al. Increased frequency of alleles associated with elevated tumor necrosis factor-alpha levels in children with Kawasaki disease. Pediatr Res. 2001;49(5):686-90. doi: 10.1203/00006450-200105000-00013. [PubMed: 11328953].

15. Siadati A, Sabouni F. Kawasaki disease (KD) in Iran: A report of 85 cases. J Compr Ped. 2007;1(2):9-12.

16. Newburger JW, Takahashi M, Gerber MA, Gewitz MH, Tani LY, Burns JC et al. Diagnosis, treatment, and long-term management of Kawasaki disease: A statement for health professionals from the Committee on Rheumatic Fever, Endocarditis, and Kawasaki Disease, Council on Cardiovascular Disease in the Young, American Heart Association. Pediatrics. 2004;114(6):1708-33. doi: 10.1542/peds.2004-2182. [PubMed: 15574639].

17. Burns IC, Cayan DR, Tong G, Bainto EV, Turner CL, Shike H, et al. Seasonality and temporal clustering of Kawasaki syndrome. Epidemiology. 2005;16(2):220-5. doi: 10.1097/01.ede.0000152901.06689.d4. [PubMed: 15703537]. [PubMed Central: PMC2894624]

18. Nikyar AR, Kordydarian R, Lranpour R. Cardiac sequelae of kawasaki disease in Isfahan, Iran. Med J Islamic Republic Iran. 2002;15(4):195-8. 